\title{
MAPT subhaplotypes in corticobasal degeneration: assessing associations with disease risk, severity of tau pathology, and clinical features
}

Rebecca R. Valentino ${ }^{1 \dagger}$, Shunsuke Koga ${ }^{1 \dagger}$, Ronald L. Walton ${ }^{1}$, Alexandra I. Soto-Beasley ${ }^{1}$, Naomi Kouri', Michael A. DeTure ${ }^{1}$, Melissa E. Murray ${ }^{1}$, Patrick W. Johnson², Ronald C. Petersen³, Bradley F. Boeve ${ }^{3}$, Ryan J. Uitti ${ }^{4}$, Zbigniew K. Wszolek ${ }^{4}$, Dennis W. Dickson ${ }^{1 *}$, Owen A. Ross ${ }^{1,5}$ and Michael G. Heckman ${ }^{2 *}$ (1)

\begin{abstract}
The microtubule-associated protein tau (MAPT) H1 haplotype is the strongest genetic risk factor for corticobasal degeneration (CBD). However, the specific $\mathrm{H} 1$ subhaplotype association is not well defined, and it is not clear whether any MAPT haplotypes influence severity of tau pathology or clinical presentation in CBD. Therefore, in the current study we examined 230 neuropathologically confirmed CBD cases and 1312 controls in order to assess associations of MAPT haplotypes with risk of CBD, severity of tau pathology (measured as semi-quantitative scores for coiled bodies, neurofibrillary tangles, astrocytic plaques, and neuropil threads), age of CBD onset, and disease duration. After correcting for multiple testing ( $P<0.0026$ considered as significant), we confirmed the strong association between the MAPT H2 haplotype and decreased risk of CBD (Odds ratio $=0.26, P=2 \times 10^{-12}$ ), and also observed a novel association between the $\mathrm{H} 1 \mathrm{~d}$ subhaplotype and an increased CBD risk (Odds ratio $=1.76, P=0.002$ ). Additionally, although not statistically significant after correcting for multiple testing, the $\mathrm{H} 1 \mathrm{c}$ haplotype was associated with a higher risk of CBD (Odds ratio $=1.49, P=0.009)$. No MAPT haplotypes were significantly associated with any tau pathology measures, age of CBD onset, or disease duration. Though replication will be important and there is potential that population stratification could have influenced our findings, these results suggest that several MAPT H1 subhaplotypes are primarily responsible for the strong association between MAPT $\mathrm{H1}$ and risk of $\mathrm{CBD}$, but that $\mathrm{H} 1$ subhaplotypes are unlikely to play a major role in driving tau pathology or clinical features. Our findings also indicate that similarities in MAPT haplotype risk-factor profile exist between CBD and the related tauopathy progressive supranuclear palsy, with $\mathrm{H} 2, \mathrm{H} 1 \mathrm{~d}$, and $\mathrm{H} 1 \mathrm{C}$ displaying associations with both diseases.
\end{abstract}

Keywords: Corticobasal degeneration, MAPT, Genetics, Neuropathology

\footnotetext{
*Correspondence: dickson.dennis@mayo.edu; heckman.michael@mayo.edu ${ }^{\dagger}$ Rebecca R. Valentino and Shunsuke Koga have contributed equally to this work

Dennis W. Dickson, Owen A. Ross and Michael G. Heckman: Joint senior authors

1 Department of Neuroscience, Mayo Clinic, 4500 San Pablo Road, Jacksonville, FL 32224, USA

${ }^{2}$ Division of Biomedical Statistics and Informatics, Mayo Clinic, 4500 San Pablo Road, Jacksonville, FL 32224, USA

Full list of author information is available at the end of the article
}

\section{Introduction}

Corticobasal degeneration (CBD) is a rare and progressive neurodegenerative disorder, with an estimated prevalence of approximately 6 cases per 100,000 people [12]. Age of disease onset is most commonly between 40 and 70 years, and the average survival time is 7 years [12]. Patients with CBD can present with a variety of clinical features which overlap with other neurodegenerative original author(s) and the source, provide a link to the Creative Commons licence, and indicate if changes were made. The images or other third party material in this article are included in the article's Creative Commons licence, unless indicated otherwise in a credit line to the material. If material is not included in the article's Creative Commons licence and your intended use is not permitted by statutory regulation or exceeds the permitted use, you will need to obtain permission directly from the copyright holder. To view a copy of this licence, visit http://creativecommons.org/licenses/by/4.0/. The Creative Commons Public Domain Dedication waiver (http://creativeco mmons.org/publicdomain/zero/1.0/) applies to the data made available in this article, unless otherwise stated in a credit line to the data. 
disorders, including progressive supranuclear palsy (PSP), Alzheimer's disease (AD), and frontotemporal dementia (FTD), which makes clinical diagnosis challenging $[2,5,12]$. Indeed, a definitive diagnosis of CBD can only be made neuropathologically, where CBD is characterized by deposition of tau in neurons and glia in the form of pretangles, neurofibrillary tangles (NFT), neuropil threads (NT), astrocytic plaques (AP), and oligodendroglial coiled bodies (CB) [7]. Tau in CBD is composed disproportionately of isoforms with four repeats in the microtubule binding domain ( $4 \mathrm{R} \mathrm{tau}$ ) in the tau gene (MAPT) [4]. While PSP and CBD are both primary 4R-tauopathies, tau pathology occurs disproportionately in forebrain structures in CBD and in hindbrain structures in PSP, although both regions are affected in both disorders $[12,18]$.

Due to its rarity, little is known about the genetics of CBD [20]. Initial studies of common genetic variation in CBD implicated the MAPT H1 haplotype [6, 10, 17, 25]. These findings were subsequently confirmed in the first genome-wide association study (GWAS) of CBD [11], where the H1 haplotype was identified as the strongest genetic risk factor (Odds ratio $[\mathrm{OR}]=3.70$, $P=1.4 \times 10^{-12}$ ), with the rs 242557 variant which partially tags the H1c subhaplotype also displaying some evidence of association with $\mathrm{CBD}$ risk ( $\left.\mathrm{OR}=1.57, P=7.9 \times 10^{-6}\right)$. The MAPT gene is characterized by two main haplotypes related to a large inversion on chromosome 17 , whereby the $\mathrm{H} 1$ haplotype is more common and the $\mathrm{H} 2$ haplotype is rarer and virtually absent for individuals of non-European backgrounds [21]. The $\mathrm{H} 1$ haplotype can be further categorized into more than 20 common subhaplotypes, including H1c and others [17]. Notably, a recent study observed associations of several MAPT subhaplotypes with risk of PSP, and to a lesser extent with severity of tau pathology in PSP [9]. The clinical and neuropathological similarities between PSP and CBD, as well as evidence that indicates that these two tauopathies have significant shared genetic risk [26], suggests that $\mathrm{H} 1$ subhaplotypes could also play a role in CBD. However, there has been no study to date of notable sample size that has examined how MAPT subhaplotypes may influence susceptibility to CBD or clinical and neuropathological features of CBD. Therefore, the aim of the current study was to examine associations of MAPT subhaplotypes with risk of CBD, tau pathology severity, and clinical features in this rare and understudied neurodegenerative disorder.

\section{Materials and methods}

\section{Study patients}

This study included a total of 230 neuropathologically-confirmed CBD cases and 1312 clinical controls. Of note, 139 of the CBD cases were included in the aforementioned CBD GWAS (72 in the discovery stage, and 67 in the replication stage) [11]. The CBD cases were obtained from the Mayo Clinic brain bank for neurodegenerative disorders between 1998 and 2019. All cases were examined by a single neuropathologist (DWD) using standardized histopathologic methods and phospho-tau immunochemistry. Diagnosis of CBD was made using current neuropathologic criteria [7]. Controls were recruited from the Mayo Clinic in Jacksonville, Florida $(\mathrm{N}=881)$ or Rochester, Minnesota $(\mathrm{N}=431)$ and were free from neurological disease. Information regarding age of $\mathrm{CBD}$ onset and disease duration was available from medical records for 188 of the 230 CBD cases. Controls were in part collected through the Mayo Clinic Study of Aging (MCSA), Alzheimer's disease Research Center (ADRC), and the Mayo Clinic Udall Parkinson's Disease Research Center of Excellence. All subjects were unrelated non-Hispanic Caucasians. Characteristics of CBD cases and controls are detailed in Table 1.

\section{Genetic analysis}

Total genomic DNA was extracted from peripheral blood lymphocytes in controls and from brain tissue in CBD cases using standard protocols [15]. A total of six MAPT variants (rs1467967, rs242557 [the H1c haplotype partial tagging variant], rs3785883, rs2471738, rs8070723 [the $\mathrm{H} 2$ haplotype tagging variant], and rs7521) were genotyped to examine MAPT haplotypes as previously described [17]. MAPT variants were genotyped using TaqMan SNP genotyping assays on an ABI 7900HT Fast Real-Time PCR system (Applied Bio systems, Foster City, CA, USA) according to manufacturer instructions (primer sequences available upon request). Genotypes were called using TaqMan Genotyper Software v1.3 (Applied Bio systems, Foster City, CA, USA). There were no deviations from Hardy-Weinberg equilibrium in controls (all $P>0.01$ after Bonferroni correction), and genotype call rates were $100 \%$ for each variant. Genotype frequencies for each variant are summarized in Additional file 1: Table S1.

\section{Neuropathological assessment}

For a subset of 196 CBD cases, a single neuropathologist (DWD) assessed semi-quantitative tau pathology measures on a $0-3$ severity scale $(0=$ none; $1=$ mild; $2=$ moderate; $3=$ severe). All sections were processed identically with phospho-tau monoclonal antibody (CP13, from Dr. Peter Davies, Feinstein Institute, Long Island, NY). Immunochemistry was performed using a DAKO Autostainer. CB, NFT, AP, and NT were evaluated and scored in 21 different neuroanatomical regions which are vulnerable to CBD pathology (Additional file 1: Table S2) [8]. For each of the four different tau pathology measures 
Table 1 Characteristics of CBD patients and controls

\begin{tabular}{|c|c|c|}
\hline Variable & CBD patients $(\mathrm{N}=230)$ & Controls $(N=1312)$ \\
\hline Age (years) $^{1}$ & $70(46,96)$ & $69(45,92)$ \\
\hline \multicolumn{3}{|l|}{ Sex } \\
\hline Female & $119(51.7 \%)$ & $611(46.6 \%)$ \\
\hline Male & $111(48.3 \%)$ & $701(53.4 \%)$ \\
\hline Age of CBD onset (years) & $64(41,86)$ & N/A \\
\hline Disease duration (years) & $6(2,16)$ & N/A \\
\hline \multicolumn{3}{|l|}{ Braak stage } \\
\hline 0 & $27(13.8 \%)$ & N/A \\
\hline 1 & $36(18.4 \%)$ & N/A \\
\hline$\|$ & $66(33.7 \%)$ & N/A \\
\hline III & $53(27.0 \%)$ & N/A \\
\hline IV & $11(5.6 \%)$ & N/A \\
\hline V & $2(1.0 \%)$ & N/A \\
\hline $\mathrm{Vl}$ & $1(0.5 \%)$ & N/A \\
\hline \multicolumn{3}{|l|}{ Thal phase } \\
\hline 0 & $105(53.6 \%)$ & N/A \\
\hline 1 & $35(17.9 \%)$ & N/A \\
\hline 2 & $24(12.2 \%)$ & N/A \\
\hline 3 & $25(12.8 \%)$ & N/A \\
\hline 4 & $3(1.5 \%)$ & N/A \\
\hline 5 & $4(2.0 \%)$ & $\mathrm{N} / \mathrm{A}$ \\
\hline CB overall tau pathology score & $0.77(0.23,1.75)$ & N/A \\
\hline NFT overall tau pathology score & $2.19(1.13,2.62)$ & N/A \\
\hline AP overall tau pathology score & $0.53(0.24,1.04)$ & N/A \\
\hline NT overall tau pathology score & $2.52(1.24,2.95)$ & N/A \\
\hline
\end{tabular}

CBD corticobasal degeneration, $C B$ coiled bodies, NFT neurofibrillary tangles, $A P$ astrocytic plaques, $N T$ neuropil threads

The sample median (minimum, maximum) is given for age. ${ }^{1}$ Age represents age at death in CBD patients and age at blood draw in controls. Information was unavailable in $\mathrm{CBD}$ cases for age of $\mathrm{CBD}$ onset $(\mathrm{N}=42)$, disease duration $(\mathrm{N}=42)$, Braak stage $(\mathrm{N}=34)$, Thal phase $(\mathrm{N}=34)$, $C B$ overall tau pathology score $(\mathrm{N}=34)$, NFT overall tau pathology score $(\mathrm{N}=34)$, AP overall tau pathology score $(\mathrm{N}=34)$, and $\mathrm{NT}$ overall tau pathology score $(\mathrm{N}=34)$

(CB, NFT, AP, and NT), one overall score was created by calculating the mean of the semi-quantitative scores $(0$, 1,2 , or 3) for each CBD case across all neuroanatomical regions, with a higher overall score indicating a greater severity of tau pathology (Table 1). In calculation of the four overall tau pathology scores, for CBD cases who did not have information available in a given region for a given tau pathology measure, these values were imputed by using the mean of the values of the CBD cases who did have this information available; this imputation was performed in order to avoid biasing overall tau pathology scores for CBD cases who did not have a tau pathology score in a neuroanatomical region (or regions) with generally less severe, or more severe, tau pathology. Any CBD cases who had missing data for greater than $50 \%$ of neuroanatomical regions for a given tau pathology measure were not included in analysis involving the overall score for that measure.

CBD cases were additionally assessed for Alzheimer type pathology with thioflavin $\mathrm{S}$ fluorescent microscopy.
Braak NFT stage [3] and Thal amyloid phase [22] were assessed for each CBD case based on the density and distribution of plaques and tangles as previously described [16].

\section{Statistical analysis}

Associations between six-variant MAPT haplotypes and risk of $\mathrm{CBD}$ were examined using score tests for association under a logistic regression framework [19], where tests were adjusted for age and sex. ORs and 95\% CIs were estimated and are interpreted as the multiplicative increase in the odds of CBD corresponding to each additional copy of the given haplotype. In analysis of CBD cases, associations of six-variant $M A P T$ haplotypes with overall $\mathrm{CB}, \mathrm{NFT}$, AP, and NT tau pathology scores, age of $\mathrm{CBD}$ onset, and disease duration were assessed using score tests for association under a linear regression framework [19]; tests were adjusted for age at death, sex, Braak stage, and Thal phase (analysis of tau pathology scores), for sex (analysis of age of CBD onset), and 
for sex and age of CBD onset (analysis of disease duration). Regression coefficients ( $\beta$ ) and 95\% CIs were estimated and are interpreted as the additive change in the mean outcome value corresponding to each additional copy of the given haplotype. Haplotypes that occurred in $<1 \%$ of subjects in a given analysis were excluded for that analysis.

We utilized a Bonferroni correction for multiple testing, after which $P$ values $<0.0026$ (19 tests) were considered as significant when examining associations with risk of CBD and with overall tau pathology scores, and $P$ values $<0.0028$ (18 tests) were considered as statistically significant when assessing associations with age of CBD onset and disease duration. All statistical tests were twosided. Statistical analyses were performed using R Statistical Software (version 3.6.2; R Foundation for Statistical Computing, Vienna, Austria).

\section{Results}

Associations between six-variant MAPT haplotypes and risk of CBD are displayed in Table 2. After correcting for the 19 haplotypes that were examined $(P<0.0026$ considered as significant), the $\mathrm{H} 2$ haplotype was strongly associated with a decreased risk of $\mathrm{CBD}(\mathrm{OR}=0.26,95 \%$ CI: $0.18-0.38, P=2 \times 10^{-12}$ ), while the H1d subhaplotype was associated with increased CBD risk $(\mathrm{OR}=1.76$, 95\% CI: $1.22-2.52, P=0.002)$. Additionally, although not quite statistically significant, the $\mathrm{H} 1 \mathrm{c}$ subhaplotype was also associated with a higher risk of $\mathrm{CBD}(\mathrm{OR}=1.49,95 \%$ CI: $1.11-2.00, P=0.009)$. Other nominally significant $(P<0.05)$ findings occurred for the H1b $(\mathrm{OR}=1.31,95 \%$ CI: $1.00-1.72, P=0.049)$, and $\mathrm{H} 1 \mathrm{i}(\mathrm{OR}=1.65,95 \% \mathrm{CI}$ : $1.01-2.70, P=0.047)$ subhaplotypes.

When evaluating associations of MAPT haplotypes with CB, NFT, AP, and NT overall tau pathology scores in CBD cases (Table 3), no associations survived correction for multiple testing. Several nominally significant findings were observed (NFT: H1p $[P=0.021]$ and $\mathrm{H} 1 \mathrm{z}$ $[P=0.033]$; AP: H1e $[P=0.007]$; NT: H1p $[P=0.028])$, however none of these involved the $\mathrm{H} 2, \mathrm{H} 1 \mathrm{~d}$, or $\mathrm{H} 1 \mathrm{c}$ haplotypes that were associated with CBD risk. Similarly, there were no statistically significant $(P<0.0028$ considered significant) associations between MAPT haplotypes and either age of CBD onset or disease duration (Table 4); the only nominally significant finding occurred for the rare H1p subhaplotype, which was associated with

Table 2 Associations between MAPT haplotypes and risk of CBD

\begin{tabular}{|c|c|c|c|c|c|c|c|c|c|c|}
\hline \multirow[t]{2}{*}{ Haplotype } & \multicolumn{6}{|c|}{ MAPT variant } & \multicolumn{2}{|c|}{ Haplotype frequency (\%) } & \multicolumn{2}{|c|}{ Association with CBD } \\
\hline & rs1467967 & rs242557 & rs3785883 & rs2471738 & rs8070723 & rs7521 & $\begin{array}{l}\text { CBD } \\
\text { patients } \\
(\mathrm{N}=230)\end{array}$ & $\begin{array}{l}\text { Controls } \\
(N=1312)\end{array}$ & OR $(95 \% \mathrm{Cl})$ & $P$ value \\
\hline $\mathrm{H} 1 \mathrm{~b}$ & G & G & G & C & A & A & 20.2 & 16.0 & $1.31(1.00,1.72)$ & 0.049 \\
\hline $\mathrm{H} 1 \mathrm{C}$ & A & A & G & $\mathrm{T}$ & A & G & 15.2 & 11.3 & $1.49(1.11,2.00)$ & 0.009 \\
\hline $\mathrm{H} 1 \mathrm{~d}$ & A & A & G & C & A & A & 10.9 & 7.1 & $1.76(1.22,2.52)$ & 0.002 \\
\hline H1e & A & G & G & C & A & A & 7.5 & 9.0 & $0.92(0.62,1.36)$ & 0.66 \\
\hline $\mathrm{H} 1 \mathrm{f}$ & G & G & $A$ & C & A & A & 0.0 & 1.2 & $\mathrm{~N} / \mathrm{A}^{1}$ & 0.14 \\
\hline $\mathrm{H} 1 \mathrm{~g}$ & G & A & $A$ & C & A & A & 1.6 & 1.1 & $1.45(0.53,3.96)$ & 0.47 \\
\hline $\mathrm{H} 1 \mathrm{~h}$ & A & G & A & C & A & A & 5.4 & 4.1 & $1.22(0.72,2.08)$ & 0.47 \\
\hline $\mathrm{H} 1 \mathrm{i}$ & G & A & G & C & $A$ & A & 5.9 & 4.4 & $1.65(1.01,2.70)$ & 0.047 \\
\hline $\mathrm{H} 1 \mathrm{I}$ & A & G & $A$ & C & $A$ & G & 3.7 & 3.0 & $1.10(0.61,1.99)$ & 0.75 \\
\hline $\mathrm{H} 1 \mathrm{~m}$ & G & A & $G$ & C & A & G & 1.6 & 2.9 & $0.77(0.36,1.64)$ & 0.50 \\
\hline H1O & A & A & A & C & A & A & 3.5 & 2.3 & $1.81(0.93,3.50)$ & 0.080 \\
\hline H1p & G & G & $G$ & $\mathrm{~T}$ & A & G & 0.9 & 1.5 & $0.82(0.28,2.43)$ & 0.72 \\
\hline $\mathrm{H} 1 \mathrm{q}$ & A & A & G & $\mathrm{T}$ & A & A & 1.3 & 1.0 & $1.49(0.57,3.89)$ & 0.42 \\
\hline $\mathrm{H} 1 \mathrm{r}$ & A & G & G & $\mathrm{T}$ & A & G & 2.6 & 1.1 & $2.03(0.83,4.93)$ & 0.12 \\
\hline $\mathrm{H} 1 \mathrm{u}$ & $A$ & A & G & C & A & G & 4.4 & 2.4 & $1.60(0.87,2.95)$ & 0.13 \\
\hline $\mathrm{H} 1 \mathrm{v}$ & G & G & A & $\mathrm{T}$ & A & G & 1.7 & 1.2 & $1.10(0.40,3.03)$ & 0.85 \\
\hline $\mathrm{H} 1 \mathrm{x}$ & G & A & A & $\mathrm{T}$ & A & G & 2.2 & 1.3 & $1.54(0.64,3.68)$ & 0.34 \\
\hline H1y & $A$ & A & A & $\mathrm{T}$ & A & G & 0.5 & 1.6 & $0.54(0.15,1.95)$ & 0.35 \\
\hline $\mathrm{H} 2$ & $A$ & G & G & C & G & G & 7.1 & 22.7 & $0.26(0.18,0.38)$ & $2 \times 10^{-12}$ \\
\hline
\end{tabular}

ORs, $95 \% \mathrm{Cls}$, and $P$ values result from score tests of association that were adjusted for age and sex. ORs correspond to each additional copy of the given haplotype. $P$ values $<0.0026$ are considered as statistically significant after applying a Bonferroni correction for multiple testing

$C B D$ corticobasal degeneration, $O R$ odds ratio, $C l$ confidence interval

1 The $\mathrm{H} 1 \mathrm{f}$ haplotype was not observed in CBD cases, and therefore estimation of an OR was not possible 


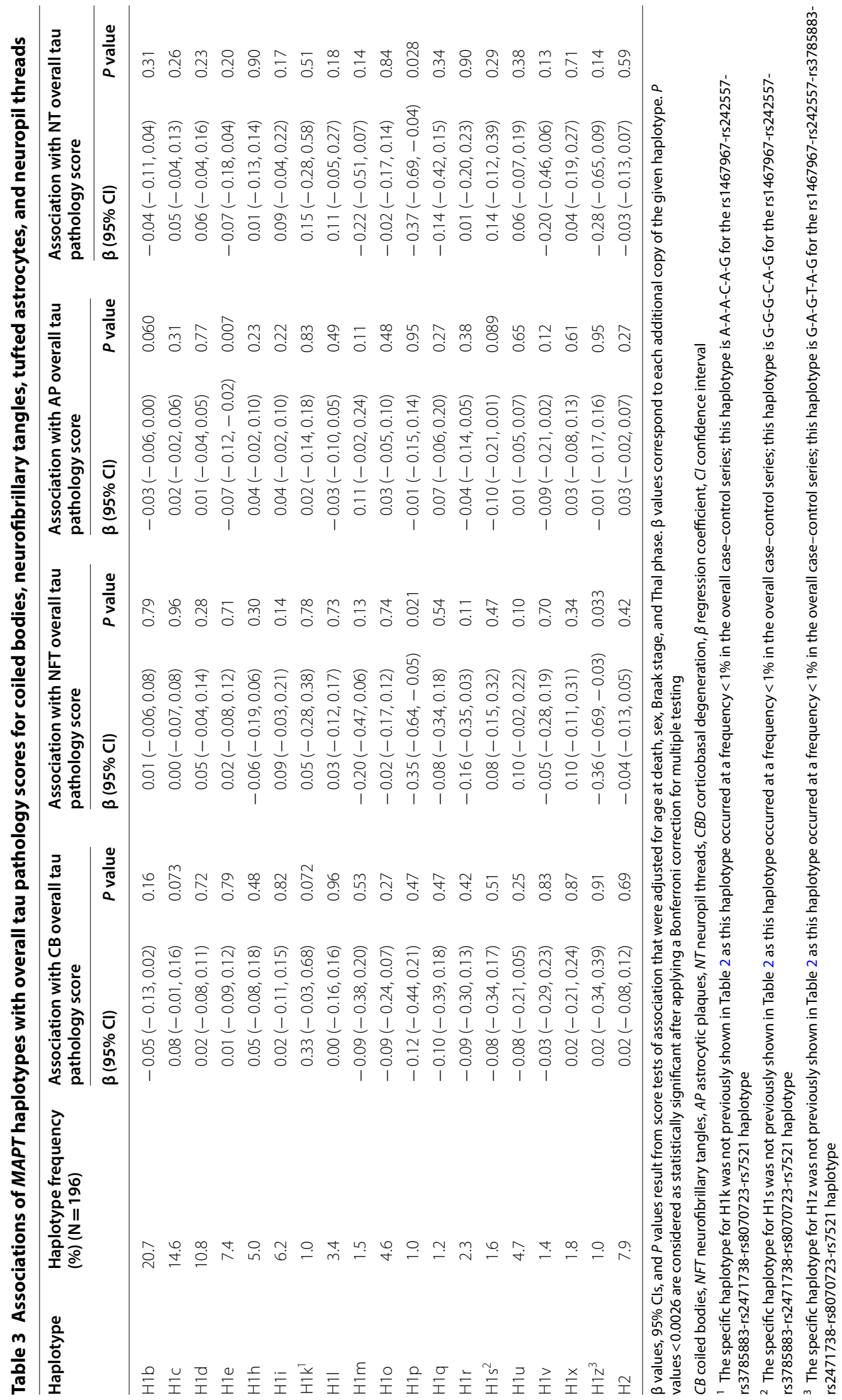


Table 4 Associations of MAPT haplotypes with age of CBD onset and disease duration

\begin{tabular}{|c|c|c|c|c|c|}
\hline \multirow[t]{2}{*}{ Haplotype } & \multirow{2}{*}{$\begin{array}{l}\text { Haplotype frequency }(\%) \\
(\mathrm{N}=188)\end{array}$} & \multicolumn{2}{|c|}{ Association with age of CBD onset } & \multicolumn{2}{|c|}{ Association with disease duration } \\
\hline & & $\beta(95 \% \mathrm{Cl})$ & $P$ value & $\beta(95 \% \mathrm{Cl})$ & $P$ value \\
\hline $\mathrm{H} 1 \mathrm{~b}$ & 21.6 & $-0.48(-2.53,1.56)$ & 0.64 & $0.10(-0.52,0.72)$ & 0.76 \\
\hline $\mathrm{H} 1 \mathrm{C}$ & 15.1 & $-1.10(-3.42,1.22)$ & 0.36 & $0.00(-0.71,0.71)$ & 0.99 \\
\hline $\mathrm{H} 1 \mathrm{~d}$ & 10.8 & $1.18(-1.51,3.88)$ & 0.39 & $0.12(-0.70,0.94)$ & 0.78 \\
\hline H1e & 6.7 & $0.05(-3.27,3.37)$ & 0.98 & $0.38(-0.63,1.38)$ & 0.46 \\
\hline $\mathrm{H} 1 \mathrm{~h}$ & 4.9 & $-1.00(-4.76,2.76)$ & 0.60 & $0.08(-1.06,1.23)$ & 0.89 \\
\hline $\mathrm{H} 1 \mathrm{i}$ & 6.3 & $-1.62(-5.20,1.96)$ & 0.38 & $-1.04(-2.12,0.04)$ & 0.061 \\
\hline $\mathrm{H} 1 \mathrm{k}^{1}$ & 1.0 & $2.49(-7.51,12.49)$ & 0.63 & $-2.60(-5.61,0.41)$ & 0.093 \\
\hline $\mathrm{H} 11$ & 3.8 & $1.80(-2.49,6.09)$ & 0.41 & $0.31(-1.00,1.62)$ & 0.64 \\
\hline $\mathrm{H} 1 \mathrm{~m}$ & 1.7 & $-4.68(-12.23,2.87)$ & 0.23 & $-2.01(-4.30,0.28)$ & 0.087 \\
\hline $\mathrm{H} 1 \mathrm{O}$ & 4.3 & $-2.72(-7.19,1.75)$ & 0.24 & $-0.48(-1.85,0.88)$ & 0.49 \\
\hline $\mathrm{H} 1 \mathrm{p}$ & 1.1 & $0.94(-7.98,9.85)$ & 0.84 & $3.42(0.76,6.08)$ & 0.013 \\
\hline $\mathrm{H} 1 \mathrm{q}$ & 1.2 & $0.08(-7.97,8.12)$ & 0.99 & $-0.06(-2.50,2.38)$ & 0.96 \\
\hline $\mathrm{H} 1 \mathrm{r}$ & 2.1 & $-0.27(-6.67,6.13)$ & 0.93 & $0.87(-1.06,2.81)$ & 0.38 \\
\hline $\mathrm{H} 1 \mathrm{~s}^{2}$ & 1.3 & $1.11(-6.92,9.13)$ & 0.79 & $0.29(-2.15,2.73)$ & 0.82 \\
\hline $\mathrm{H} 1 \mathrm{u}$ & 4.4 & $2.56(-1.28,6.41)$ & 0.19 & $0.01(-1.17,1.19)$ & 0.98 \\
\hline H1v & 1.3 & $0.42(-7.03,7.87)$ & 0.91 & $1.79(-0.46,4.03)$ & 0.12 \\
\hline $\mathrm{H} 1 \mathrm{x}$ & 1.9 & $0.66(-5.57,6.90)$ & 0.84 & $-0.20(-2.09,1.69)$ & 0.84 \\
\hline $\mathrm{H} 2$ & 7.7 & $1.23(-1.58,4.04)$ & 0.39 & $-0.02(-0.87,0.84)$ & 0.97 \\
\hline
\end{tabular}

$\beta$ values, $95 \% \mathrm{Cls}$, and $P$ values result from score tests of association that were adjusted for sex (age of CBD onset analysis) or sex and age of CBD onset (disease duration analysis). $\beta$ values correspond to each additional copy of the given haplotype. $P$ values $<0.0028$ are considered as statistically significant after applying a Bonferroni correction for multiple testing

$C B D$ corticobasal degeneration, $\beta$ regression coefficient, $C l$ confidence interval

1 The specific haplotype for $\mathrm{H} 1 \mathrm{k}$ was not previously shown in Table 2 as this haplotype occurred at a frequency $<1 \%$ in the overall case-control series; this haplotype is A-A-A-C-A-G for the rs1467967-rs242557-rs3785883-rs2471738-rs8070723-rs7521 haplotype

2 The specific haplotype for $\mathrm{H} 1 \mathrm{~s}$ was not previously shown in Table 2 as this haplotype occurred at a frequency $<1 \%$ in the overall case-control series; this haplotype is G-G-G-C-A-G for the rs1467967-rs242557-rs3785883-rs2471738-rs8070723-rs7521 haplotype

a longer disease duration $(\beta=3.42$, 95\% CI: $0.76-6.08$, $P=0.013)$.

\section{Discussion}

Although there is still much to be understood regarding genetic risk factors for CBD, findings to date have demonstrated a strong association involving the MAPT H1 haplotype [11]. Examination of MAPT H1 subhaplotypes provides the opportunity to better understand any notable association involving the $\mathrm{H} 1$ haplotype, and indeed several studies have observed associations between MAPT $\mathrm{H} 1$ subhaplotypes and various neurodegenerative diseases $[1,9,13,14,17,24]$. Herein, in addition to further confirming the strong association between the $\mathrm{H} 2$ haplotype and reduced risk of CBD, we have identified a novel association between the H1d subhaplotype and risk of $\mathrm{CBD}$. The H1c subhaplotype also displayed some degree of association with CBD, though this was not significant after multiple testing correction (possibly due to lack of power given the relatively small sample size). No strong associations of MAPT H1 subhaplotypes with severity of tau pathology, age of CBD onset, or disease duration were noted. Though replication will be crucial, these findings suggest that several specific MAPT H1 subhaplotypes (H1d and H1c) are primarily responsible for the strong association between MAPT $\mathrm{H} 1$ and risk of $\mathrm{CBD}$, but that $\mathrm{H} 1$ subhaplotypes are unlikely to play a major role in driving severity of tau pathology or clinical features.

Only one previous study has assessed associations between MAPT subhaplotypes and risk of CBD, where Pittman et al. [17] examined a series of 44 CBD cases and 131 controls. Their results are generally in line with the findings of our current study; $\mathrm{H} 2$ was associated with a significantly decreased risk of CBD $(8.2 \%$ vs. $22.0 \%$, $P=0.020$ ), and although not statistically significant in this small series with very low power to detect associations, both H1c $(17.7 \%$ vs. $7.8 \%, P=0.066)$ and H1d ( $7.5 \%$ vs. $4.0 \%, P=0.49$ ) were observed more frequently in CBD cases than controls. The authors also observed a nominally significant association between $\mathrm{H} 1 \mathrm{n}$ and a decreased risk of CBD $(0.0 \%$ vs. $4.3 \%, P=0.018)$. The H1n haplotype was observed in only $0.6 \%$ of all subjects 
in our case-control series and therefore was not formally assessed in disease-association analysis where only haplotypes that were observed at a frequency of at least $1 \%$ in the overall series were assessed. Further examination of the H1n subhaplotype in our series reveals a frequency of $0.0 \%$ in CBD cases and $0.6 \%$ in controls, which does not replicate the previously observed nominally significant finding. Overall, the results of the study by Pittman et al. support those of our current study, which implicates the H1c and H1d MAPT H1 subhaplotypes in susceptibility to CBD.

The results of our disease-association haplotype analysis are somewhat in line with those of a previous study by our group involving PSP [9], which is not surprising given the similar clinical and neuropathological features of CBD and PSP. More specifically, with a sample size of 802 PSP cases and 1312 controls (the same control group examined in the current study), the MAPT H2 haplotype was more strongly associated with PSP $(\mathrm{OR}=0.16$, $\left.P=7 \times 10^{-49}\right)$ than CBD $\left(\mathrm{OR}=0.26, P=2 \times 10^{-12}\right)$, H1d was significantly associated with an increased risk of PSP $\left(\mathrm{OR}=1.86, P=2 \times 10^{-6}\right)$ with a similar effect size compared to $\mathrm{CBD}(\mathrm{OR}=1.76, P=0.002)$, while $\mathrm{H} 1 \mathrm{c}$ was also more strongly associated with PSP risk $(\mathrm{OR}=2.15$, $\left.P=2 \times 10^{-14}\right)$ than it was with $\mathrm{CBD} \quad(\mathrm{OR}=1.49$, $P=0.009)$. Additionally, H1o was associated with a significantly higher risk of PSP $\left(\mathrm{OR}=2.60, P=2 \times 10^{-5}\right)$, which is not overly divergent from the non-significant $\mathrm{OR}$ of $1.81(P=0.080)$ that we noted for CBD; the absence of a statistically significant association between MAPT H1o and risk of CBD in our study may be due to the less precise haplotype frequencies and lower power to detect associations that we had in comparison to our previous PSP study where the number of cases was greater than threefold higher. Though weaker evidence, the H1i subhaplotype was nominally associated with increased risk in both CBD $(\mathrm{OR}=1.65, P=0.047)$ and PSP $(\mathrm{OR}=1.56$, $P=0.02)$. Conversely, the H1g subhaplotype was a significant risk factor for PSP (OR=3.64, $\left.P=2 \times 10^{-6}\right)$ but displayed very little evidence of an association with CBD $(\mathrm{OR}=1.45, P=0.47)$, while $\mathrm{H} 1 \mathrm{~b}$ was nominally associated with $\mathrm{CBD}(\mathrm{OR}=1.31, P=0.049)$ but not PSP $(\mathrm{OR}=1.06, P=0.57)$. Larger series will be needed to further confirm the suggestive association between $\mathrm{H} 1 \mathrm{c}$ and CBD that we observed, as well as to examine whether the H1o subhaplotype may be a genetic risk factor for CBD as it is for PSP. Overall, our findings suggest that although similarities in MAPT haplotype risk factor profile exist between these two primary 4R-tauopathies, with $\mathrm{H} 2$, $\mathrm{H} 1 \mathrm{~d}$, and H1c displaying associations with both diseases, the strength of association may be stronger for PSP as evidenced by generally weaker association ORs in CBD for the three aforementioned shared susceptibility haplotypes, and a lack of strong association with CBD for several PSP risk haplotypes ( $\mathrm{H} 1 \mathrm{~g}$ and $\mathrm{H} 1 \mathrm{o}$ ).

As previously mentioned, in the previous CBD GWAS, there was suggestive evidence of an association between the rs242557 variant that partially tags the MAPT H1c haplotype $\left(\mathrm{OR}=1.57, P=7.9 \times 10^{-6}\right)$ [11]. Interestingly, though our haplotype-specific analysis mostly supports this finding as the rs242557 risk allele (A) is observed for the H1c, H1d, H1i, and H1o subhaplotypes (all of which were associated with an increased risk of $\mathrm{CBD}$ with a $P$ value $<0.10$ ), findings differ for the H1b haplotype. H1b is the most common $\mathrm{H} 1$ subhaplotype and is associated with the protective $(G)$ allele of rs242557; the nominally significant evidence of an association between $\mathrm{H} 1 \mathrm{~b}$ and an increased risk of CBD that we observed demonstrates that rs 242557 is not consistently associated with CBD risk, and underscores the importance of assessing specific $\mathrm{H} 1$ subhaplotypes when examining the role of $M A P T$ in susceptibility to CBD.

Interestingly, despite the somewhat similar findings regarding the role of MAPT haplotypes in susceptibility to CBD and PSP, findings differ regarding associations with severity of tau pathology. This is most evident when considering the three shared protective $(\mathrm{H} 2)$ and risk (H1c and H1d) haplotypes between these two tauopathies; all three of these haplotypes were correlated with severity of tau pathology in PSP cases in the aforementioned previous study by our group, where small but nominally significant associations were observed [9]. Conversely, the MAPT H2, H1c, and H1d haplotypes were not significantly associated with severity of tau pathology in our current study of CBD cases. Of course, one explanation for this could be the aforementioned much lower power we had in this study to detect associations with severity of tau pathology in CBD. Correspondingly, it may be noteworthy that $\mathrm{H} 1 \mathrm{c}$ was associated with severity of $\mathrm{CB}$ tau pathology with a p-value of 0.073 and an effect size of 0.08 that is larger than any of those observed in PSP where nominal significance was reached for this haplotype (effect sizes of 0.05 were observed for severity of both $\mathrm{CB}$ and tufted astrocytes tau pathology). Along these lines, the smaller degree of variability in overall tau pathology scores (NT in particular) that we observed for CBD in comparison to PSP [9] would also have limited power to detect associations with these measures. Nonetheless, it is clear that regardless of the presence or absence of any statistically significant findings, no MAPT haplotypes are strongly associated with neuropathologic severity of disease in CBD or PSP. Additionally, our current findings provide further support for the hypothesis that $\mathrm{H} 1$ subhaplotypes are not major 
disease modifiers in CBD, as no significant associations with age of CBD onset or disease duration were observed.

Several limitations of our study are important to bear in mind. First, as previously referred to, the sample size is relatively small, and therefore the possibility of a type II error (i.e. false-negative finding) is important to consider, particularly for rarer haplotypes and after multiple testing adjustment. Second, without available genome-wide control markers that would have allowed us to adjust regression models for top principal components, we cannot exclude the possibility that population stratification could have influenced our findings. Finally, our study did not include a replication series, and as a result validation of our findings will be important. However, it is worth noting that the haplotype frequencies observed in our series of 1312 controls are similar to those seen in a large independent control cohort of 8144 European-American subjects from the Alzheimer's Disease Genetics Consortium (ADGC) in a previous study by Allen et al [1]. Specifically, when comparing our 1312 Mayo Clinic controls to those 8144 ADGC controls, similar haplotype frequencies were observed for $\mathrm{H} 2$ (22.7\% vs. $23.6 \%)$, H1d (7.1\% vs. $7.9 \%)$, and to a slightly lesser extent H1c (11.3\% vs. $13.2 \%)$.

\section{Conclusions}

Due to the rare nature of CBD, meta-analytic studies will be needed to gain a solid understanding of genetic risk factors. In addition to variation at $M A P T$, the initial CBD GWAS highlighted lnc-KIF13B-1 as well as possibly $M O B P$ and $S O S 1$ as susceptibility loci. Additionally, findings of a recent study suggest that mitochondrial genomic background may be associated with risk of CBD [23]. The results of our current study add to the understanding of the genetic etiology of this rare tauopathy by shedding further light on the strongest genetic risk factor for CBD that has been observed thus far-the MAPT H1 haplotype. Specifically, our results suggest that this association is driven primarily by the H1d and H1c haplotypes, but that MAPT haplotypes likely do not play a major role in clinical or neuropathologic presentation of CBD. Future replication studies that directly account for population stratification will be important to confirm our initial findings.

\section{Supplementary Information}

The online version contains supplementary material available at https://doi. org/10.1186/s40478-020-01097-z.

Additional file 1 Supplemental materials.

\section{Abbreviations}

CBD: Corticobasal degeneration; PSP: Progressive supranuclear palsy; AD: Alzheimer's disease; FTD: Frontotemporal dementia; NFT: Neurofibrillary tangles; NT: Neuropil threads; AP: Astrocytic plaques; CB: Oligodendroglial coiled bodies; 4R: Four-repeat tau isoform; MAPT: Microtubule-associated protein tau; GWAS: Genome-wide association study; MCSA: Mayo Clinic Study of Aging; ADRC: Alzheimer's disease Research Center; OR: Odds ratio; Cl: Confidence interval.

\section{Acknowledgements}

We would like to thank all those who have contributed to our research, including Dr. Peter Davies, Feinstein Institute, Long Island, NY for his invaluable tau antibodies, and particularly the patients and families who donated brain, blood and DNA samples for this work. We would like to acknowledge the continuous commitment, technical support and teamwork offered by Linda G. Rousseau, Virginia R. Phillips, and Monica Castanedes-Casey. This work was supported in part by; the Mayo Clinic Florida Tau Center WithOut Walls (NINDS U54-NS100693); an American Parkinson Disease Association (APDA), Mayo Clinic Information and Referral Center, and an APDA Center for Advanced Research. Samples included in this study were clinical controls or brain donors to the brain bank at Mayo Clinic in Jacksonville which is supported by CurePSP and the Tau Consortium.

\section{Authors' contributions}

RRV performed genotyping and quality control assessments on all samples, and assisted in drafting the manuscript. SK and DWD provided brain tissue samples for all cases and provided manuscript improvements. DWD also performed all neuropathological assessments of CBD cases. RLW provided training for genotyping methods and prepared genomic DNA extractions from provided tissues. AISB, NK, MAD, MEM, and PJW provided manuscript improvements. RCP, BFB, RJU, and ZKW recruited clinical patients and organized blood collections and provided manuscript improvements. OAR lead the study and oversaw all methodological developments and approved the final manuscript. MGH performed all statistical analysis and drafted the manuscript. All authors read and approved the final manuscript.

\section{Funding}

OAR and DWD are both supported by NINDS Tau Center without Walls Program (U54-NS100693) and NIH (UG3-NS104095). OAR is supported by NIH (P50-NS072187; R01- NS078086; U54-NS100693; U54- NS110435), DOD (W81XWH-17-1-0249) The Michael J. Fox Foundation, The Little Family Foundation, the Mayo Clinic Foundation, and the Center for Individualized Medicine. DWD receives research support from the NIH (P50-AG016574; U54-NS100693; P01-AG003949), CurePSP, the Tau Consortium, and the Robert E. Jacoby Professorship. ZKW is partially supported by the Mayo Clinic Center for Regenerative Medicine, the gifts from The Sol Goldman Charitable Trust, and the Donald G. and Jodi P. Heeringa Family, the Haworth Family Professorship in Neurodegenerative Diseases fund, and The Albertson Parkinson's Research Foundation. SK is supported by a post-doctoral fellowship from the Karin \& Sten Mortstedt $\mathrm{CBD}$ Solutions $\mathrm{AB}$. The funding organizations and sponsors had no role in any of the following: design and conduct of the study; collection, management, analysis, and interpretation of the data; preparation, review, or approval of the manuscript; and decision to submit the manuscript for publication.

\section{Availability of data and materials}

The datasets generated and/or analyzed during the current study are available from the corresponding author on reasonable request.

\section{Ethics approval}

This study was approved by the Mayo Clinic Institutional Review Board. All subjects or legal next of kin provided written informed consent.

Consent for publication

Not applicable.

\section{Competing interests}

ZKW serves as Mayo Clinic Florida PI Biogen, Inc. (228PD201), Biohaven Pharmaceuticals, Inc. (BHV4157-206 and BHV3241-301), and Neuraly, Inc. (NLY01-PD-1) grants. He serves as Co-PI of the Mayo Clinic American Parkinson Disease Association Center for Advanced Research. All other authors declare that they have no competing interests. 


\section{Author details}

1 Department of Neuroscience, Mayo Clinic, 4500 San Pablo Road, Jacksonville, FL 32224, USA. ${ }^{2}$ Division of Biomedical Statistics and Informatics, Mayo Clinic, 4500 San Pablo Road, Jacksonville, FL 32224, USA. ${ }^{3}$ Department of Neurology, Mayo Clinic, Rochester, MN, USA. ${ }^{4}$ Department of Neurology, Mayo Clinic, Jacksonville, FL, USA. ${ }^{5}$ Department of Clinical Genomics, Mayo Clinic, Jacksonville, FL, USA

Received: 26 October 2020 Accepted: 26 November 2020 Published online: 07 December 2020

\section{References}

1. Allen M, Kachadoorian M, Quicksall Z, Zou F, Chai HS, Younkin C, Crook JE, Pankratz VS, Carrasquillo MM, Krishnan S et al (2014) Association of MAPT haplotypes with Alzheimer's disease risk and MAPT brain gene expression levels. Alzheimers Res Ther 6:39. https://doi.org/10.1186/alzrt268

2. Alster P, Krzyżanowska E, Koziorowski D, Szlufik S, Różański D, Noskowska J, Mianowicz J, Michno A, Królicki L, Friedman A (2018) Difficulties in the diagnosis of four repeats (4R) tauopathic parkinsonian syndromes. Neurol Neurochir Pol 52:459-464. https://doi.org/10.1016/j.pjnns.2018.06.002

3. Braak H, Braak E (1991) Neuropathological stageing of Alzheimer-related changes. Acta Neuropathol 82:239-259. https://doi.org/10.1007/bf003 08809

4. de Silva R, Lashley T, Strand C, Shiarli AM, Shi J, Tian J, Bailey KL, Davies P, Bigio EH, Arima K et al (2006) An immunohistochemical study of cases of sporadic and inherited frontotemporal lobar degeneration using 3R- and 4R-specific tau monoclonal antibodies. Acta Neuropathol 111:329-340. https://doi.org/10.1007/s00401-006-0048-x

5. Deutschländer AB, Ross OA, Dickson DW, Wszolek ZK (2018) Atypical parkinsonian syndromes: a general neurologist's perspective. Eur J Neurol 25:41-58. https://doi.org/10.1111/ene.13412

6. Di Maria E, Tabaton M, Vigo T, Abbruzzese G, Bellone E, Donati C, Frasson E, Marchese R, Montagna P, Munoz DG et al (2000) Corticobasal degeneration shares a common genetic background with progressive supranuclear palsy. Ann Neurol 47:374-377. https://doi.org/10.1002/15318249(200003)47:3\%3c374:aid-ana15\%3e3.3.co;2-\%23

7. Dickson DW, Bergeron C, Chin SS, Duyckaerts C, Horoupian D, Ikeda K, Jellinger K, Lantos PL, Lippa CF, Mirra SS et al (2002) Office of Rare Diseases neuropathologic criteria for corticobasal degeneration. J Neuropathol Exp Neurol 61:935-946. https://doi.org/10.1093/inen/61.11.935

8. Dickson DW, Hauw J-J, Agid Y, Litvan I (2011) Progressive supranuclear palsy and corticobasal degeneration. In: Dickson DW, Weller RO (eds) Neurodegeneration: the molecular pathology of dementia and movement disorders, 2nd edn. Wiley-Blackwell, Chichester

9. Heckman MG, Brennan RR, Labbe C, Soto Al, Koga S, DeTure MA, Murray ME, Petersen RC, Boeve BF, van Gerpen JA et al (2019) Association of MAPT Subhaplotypes With Risk of Progressive Supranuclear Palsy and Severity of Tau Pathology. JAMA Neurol. https://doi.org/10.1001/jaman eurol.2019.0250

10. Houlden H, Baker M, Morris HR, MacDonald N, Pickering-Brown S, Adamson J, Lees AJ, Rossor MN, Quinn NP, Kertesz A et al (2001) Corticobasal degeneration and progressive supranuclear palsy share a common tau haplotype. Neurology 56:1702-1706. https://doi.org/10.1212/ wnl.56.12.1702

11. Kouri N, Ross OA, Dombroski B, Younkin CS, Serie DJ, Soto-Ortolaza A Baker M, Finch NCA, Yoon H, Kim J et al (2015) Genome-wide association study of corticobasal degeneration identifies risk variants shared with progressive supranuclear palsy. Nat Commun 6:7247. https://doi. org/10.1038/ncomms8247

12. Kouri N, Whitwell JL, Josephs KA, Rademakers R, Dickson DW (2011) Corticobasal degeneration: a pathologically distinct 4R tauopathy. Nat Rev Neurol 7:263-272. https://doi.org/10.1038/nrneurol.2011.43

13. Labbé C, Heckman MG, Lorenzo-Betancor O, Murray ME, Ogaki K, Soto-Ortolaza Al, Walton RL, Fujioka S, Koga S, Uitti RJ et al (2016) MAPT haplotype diversity in multiple system atrophy. Parkinsonism Relat Disorders 30:40-45. https://doi.org/10.1016/j.parkreldis.2016.06.010

14. Labbé C, Heckman MG, Lorenzo-Betancor O, Soto-Ortolaza Al, Walton RL, Murray ME, Allen M, Uitti RJ, Wszolek ZK, Smith GE et al (2016) MAPT haplotype H1G is associated with increased risk of dementia with Lewy bodies. Alzheimer's Dement J Alzheimer's Assoc 12:1297-1304. https:// doi.org/10.1016/j.jalz.2016.05.002

15. Labbé C, Soto-Ortolaza Al, Rayaprolu S, Harriott AM, Strongosky AJ, Uitti RJ, Van Gerpen JA, Wszolek ZK, Ross OA (2013) Investigating the role of FUS exonic variants in essential tremor. Parkinsonism Relat Disorders 19:755-757. https://doi.org/10.1016/j.parkreldis.2013.03.005

16. Murray ME, Lowe VJ, Graff-Radford NR, Liesinger AM, Cannon A, Przybelski SA, Rawal B, Parisi JE, Petersen RC, Kantarci Ket al (2015) Clinicopathologic and 11C-Pittsburgh compound B implications of Thal amyloid phase across the Alzheimer's disease spectrum. Brain J Neurol 138:1370-1381. https://doi.org/10.1093/brain/awv050

17. Pittman AM, Myers AJ, Abou-Sleiman P, Fung HC, Kaleem M, Marlowe L, Duckworth J, Leung D, Williams D, Kilford L et al (2005) Linkage disequilibrium fine mapping and haplotype association analysis of the tau gene in progressive supranuclear palsy and corticobasal degeneration. J Med Genet 42:837-846. https://doi.org/10.1136/jmg.2005.031377

18. Rösler TW, Tayaranian Marvian A, Brendel M, Nykänen NP, Höllerhage M, Schwarz SC, Hopfner F, Koeglsperger T, Respondek G, Schweyer K et al (2019) Four-repeat tauopathies. Prog Neurobiol 180:101644. https://doi. org/10.1016/j.pneurobio.2019.101644

19. Schaid DJ, Rowland CM, Tines DE, Jacobson RM, Poland GA (2002) Score tests for association between traits and haplotypes when linkage phase is ambiguous. Am J Hum Genet 70:425-434. https://doi.org/10.1086/33868

20. Scholz SW, Bras J (2015) Genetics underlying atypical parkinsonism and related neurodegenerative disorders. Int J Mol Sci 16:24629-24655. https ://doi.org/10.3390/ijms161024629

21. Stefansson $H$, Helgason $A$, Thorleifsson $G$, Steinthorsdottir $V$, Masson $G$, Barnard J, Baker A, Jonasdottir A, Ingason A, Gudnadottir VG et al (2005) A common inversion under selection in Europeans. Nat Genet 37:129-137. https://doi.org/10.1038/ng1508

22. Thal DR, Rüb U, Orantes M, Braak H (2002) Phases of A beta-deposition in the human brain and its relevance for the development of AD. Neurology 58:1791-1800. https://doi.org/10.1212/wnl.58.12.1791

23. Valentino RR, Tamvaka N, Heckman MG, Johnson PW, Soto-Beasley Al, Walton RL, Koga S, Uitti RJ, Wszolek ZK, Dickson DW et al (2020) Associations of mitochondrial genomic variation with corticobasal degeneration, progressive supranuclear palsy, and neuropathological tau measures. Acta Neuropathol Commun 8:162. https://doi.org/10.1186/s40478-02001035-z

24. Vandrovcova J, Pittman AM, Malzer E, Abou-Sleiman PM, Lees AJ, Wood NW, de Silva R (2009) Association of MAPT haplotype-tagging SNPs with sporadic Parkinson's disease. Neurobiol Aging 30:1477-1482. https://doi. org/10.1016/j.neurobiolaging.2007.11.019

25. Webb A, Miller B, Bonasera S, Boxer A, Karydas A, Wilhelmsen KC (2008) Role of the tau gene region chromosome inversion in progressive supranuclear palsy, corticobasal degeneration, and related disorders. Arch Neurol 65:1473-1478. https://doi.org/10.1001/archneur65.11.1473

26. Yokoyama JS, Karch CM, Fan CC, Bonham LW, Kouri N, Ross OA, Rademakers R, Kim J, Wang Y, Höglinger GU et al (2017) Shared genetic risk between corticobasal degeneration, progressive supranuclear palsy, and frontotemporal dementia. Acta Neuropathol 133:825-837. https://doi. org/10.1007/s00401-017-1693-y

\section{Publisher's Note}

Springer Nature remains neutral with regard to jurisdictional claims in published maps and institutional affiliations. 